\title{
Vigilantismus als politische Gewalt. Eine Typologie Vigilantism as Political Violence. A Typology
}

\section{Thomas Schmidt-Lux}

\section{Abstract:}

Vigilantism is usually perceived as a form of political violence. But we can distinguish three types of vigilantism with respect to its relation to the state: a) vigilantism in place of the state, b) vigilantism as a better state, and c) vigilantism beyond the state. I shall show that the relation between vigilantes and the state is thus not always oppositional, and that the political dimension of vigilante actions in fact varies considerably.

Thomas Schmidt-Lux (Ph.D.) works as a Scientific Assistant at the Institute for the Scientific Study of Culture at the University of Leipzig. He studied Sociology and History and is interested in the Sociology of Vigilantism and the Sociology of Law. E-Mail: schmidt.lux@uni-leipzig.de

tornangemeldet: 85.178.18.244:
Keywords, dt.: Vigilantismus, politische Gewalt, Staat, Stellvertretung

Keywords, engl.: vigilantism, political violence, state, agency

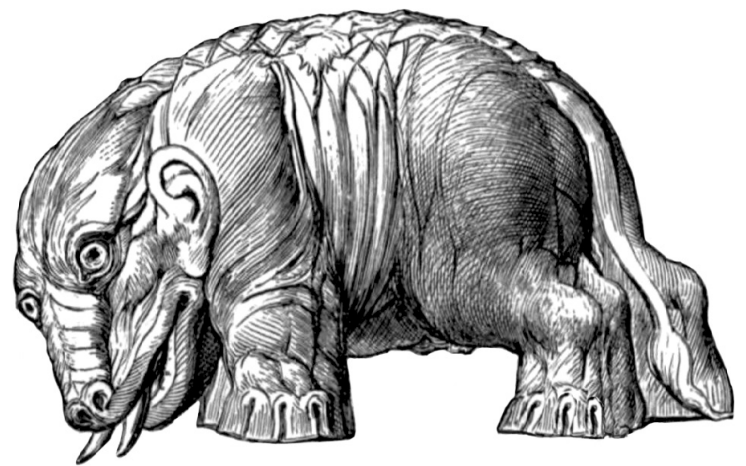




\section{Einleitung}

Unter dem Begriff ,Vigilantismus“ befasst sich seit den 1970er Jahren die Forschung mit einem Phänomenen, das im Deutschen umgangssprachlich meist als ,Selbstjustiz‘ bezeichnet wird. Man fasst darunter Situationen, in denen Menschen das Recht in die eigenen Hände nehmen, eigenmächtig Gewalt gegenüber Dritten anwenden, um gegen diese vorzugehen oder um eigene Anliegen durchzusetzen.

Zwar stehen beim Vigilantismus das Bestrafen und die Idee, Gerechtigkeit herzustellen im Vordergrund, doch gibt es auch eine Reihe politischer Implikationen. Diese sind mehr oder minder explizit, im Grunde jedoch integraler Bestandteil des Phänomens, denn allein der Verstoß gegen das staatliche Gewaltmonopol hat eine politische Dimension. Umso bemerkenswerter ist, dass die politische Seite des Vigilantismus bislang kaum systematisch diskutiert wurde. Hier setzt der folgende Beitrag an, in dem er nach dem politischen Gehalt des Vigilantismus fragt und das Phänomen mit Blick auf das Konzept der politischen Gewalt diskutiert.

Dabei ist die Frage, ob der Vigilantismus überhaupt eine Spielart politischer Gewalt darstellt, alles andere als eindeutig zu beantworten. Zwar listet das „Internationale Handbuch der Gewaltforschung“ den Eintrag zum Vigilantismus in der Rubrik „Politisch motivierte Gewalt“ (vgl. Heitmeyer et al. 2002). Doch in etlichen anderen Texten wird eine solche Verbindung nicht gezogen (vgl. Johnston 1996). Obwohl in den letzten Jahren etliche Fallstudien zum Thema entstanden, sind theoretisch-systematische Überlegungen selten. Dies soll hier nachgeholt werden. Nach wie vor ist der Begriff selbst vage, unter Vigilantismus wird vieles gefasst, was sich nur durch einen ungefähren Kern bestimmen lässt. Gleiches gilt auch für die Frage nach der Organisiertheit des Vigilantismus. Mitunter wird diese schnell negativ beantwortet. Vor allem mit Blick auf kurzfristige bzw. spontane Aktionen wie das Lynchen mutmaßlicher Diebe wird der Vigilantismus als kurzlebiges und eher unorganisiertes Phänomen beschrieben (vgl. Kowalewski 2002, 427). Doch auch an dieser Diagnose sind Zweifel angebracht.

Beiden Fragen - nach der Organisiertheit des Vigilantismus und seiner Nähe zur politischen Gewalt - soll im Folgenden systematisch nachgegangen werden. Um dabei aber nicht unvermittelt mit der ganzen Breite vigilanter Phänomene konfrontiert zu werden, muss das Feld zuvor strukturiert und differenziert werden. Dies erfolgt mittels einer Typologie, bei der drei Spielarten des Vigilan- 
tismus unterschieden werden. Die Typologie orientiert sich am jeweiligen Verhältnis der Vigilanten zum Staat. Die dabei gewonnenen Unterscheidungen und theoretischen Einsichten sollen helfen, präziser die zuvor genannten Probleme diskutieren zu können.

\section{Was ist Vigilantismus?}

Was genau ist eigentlich unter Vigilantismus zu verstehen? Im Kern meint dieser Begriff die jenseits der staatlich zugelassenen Möglichkeiten - gewaltsame bzw. unter Androhung von Gewalt erfolgende Bestrafung, Erzwingung oder Verhinderung eines abgelehnten bzw. erwünschten Handelns Anderer durch nicht-staatliche bzw. private Akteure. Gewaltförmiges oder mindestens mit Gewalt drohendes Handeln ist eine zentrale Dimension all der Beispiele, die in der gängigen Forschung unter Vigilantismus gefasst werden, und sie ist auch Bestandteil der Begriffsbestimmungen einer Reihe anderer Autoren (vgl. Abrahams 1998, 9; Johnston 1996, 226; Kowalewski 2002). Wichtig ist zudem der Umstand, dass es vigilanten Akteuren immer um das Verhalten Anderer geht, das aus ihrer Perspektive als ,abweichend‘ zu verurteilen, zu verhindern oder zu bestrafen ist: „Vigilantism arises when some established order is perceived to be under threat from the transgression (or potential transgression) of institutionalized norms“ (Johnston 1996, 229).[1]

Mit dieser Definition wird zunächst klar, dass nicht jede Form der Gewaltanwendung und nicht alles, was als Kriminalität verhandelt wird, unter Vigilantismus fällt. So ist etwa ein Banküberfall oder ein Mord aus Habgier kaum als Vigilantismus zu verstehen, da es hier primär um Materielles und um Eigeninteresse geht. Beim Vigilantismus dagegen geht es primär um die Steuerung von Anderen, um das richtige Verhalten, um die Bestrafung falschen Verhaltens, um Ordnung, um Recht. Vigilantismus ist somit eine stark normative Spielart von Gewalt: „Vigilantes typically lay claim to the moral high ground as the guardians of society. Their enemies are not simply rivals; they are also evil“ (Abrahams 1998, 78).

Wie verhält es sich nun mit der politischen Dimension des Vigilantismus? Bei manchen Autoren steht sie im Vordergrund, etwa bei Jon Rosenbaum und Peter Sederberg, deren Arbeiten sich vor allem auf die ordnungspolitischen Implikationen des Vigilantismus konzentrierten. Rosenbaum und Sederberg sehen das Aufkommen von Vigilantismus als Reaktion auf eine Bedrohung der „sozio-politischen Ordnung “ und rücken ihn daher in unmittelbare Nähe zu politischer Gewalt (Rosenbaum/
[1] Ich verzichte auf den Begriff der Devianz, weil dieser insbesondere bei Kowalewski etwas unglücklich als eine Art objektives Faktum verhandelt wird (vgl. dazu ausführlicher Schmidt-Lux 2012). 
Sederberg 1976, 7). Für viele Beispiele des Vigilantismus ist dieser Zusammenhang in der Tat unbestreitbar. Dies gilt etwa für politisch-rassistische Gruppen wie den Ku-Klux-Klan oder paramilitärische Gruppen in Lateinamerika (Abrahams 1998, 95ff.; Jones 2004). Solche Akteure verfolgen nicht nur sporadisch einzelne Personen, sondern verbinden mit ihrem Handeln dezidiert politische Forderungen bzw. Machtansprüche. Allerdings ist diese explizite politische Dimension nicht allen vigilanten Phänomenen eigen. Ebenfalls sehr heterogen ist der Organisationsgrad vigilanter Akteure. Hier reicht das Feld vom sporadisch auftretenden Einzelgänger, der auf eigene Faust mutmaßliche Kriminelle bestraft, bis hin zu dauerhaft bestehenden, klar strukturierten gewalttätigen Gruppen, die über lange Zeiträume hinweg die Kontrolle über ganze Landesteile ausüben.

Vor dem Hintergrund dieser empirischen Vielfalt wird im Folgenden eine Typologie entwickelt, die drei Arten von Vigilantismus unterscheidet. Das Kriterium, entlang dessen die Typen entwickelt werden, ist das jeweilige Verhältnis zum Staat. Dies begründet sich aus einem oben genannten, zentralen Merkmal des Vigilantismus: eine Gewaltausübung nichtstaatlicher Akteure zu sein. Zugleich macht dies seinen besonderen ,Reiz', seine spezifische Provokation und Bedeutung gerade in der Moderne aus; ist diese doch nicht zuletzt durch die Existenz von Staaten gekennzeichnet, die als eines ihrer wesentlichen Merkmale den alleinigen Zugriff auf alle Gewalt beanspruchen (vgl. Weber 1972, 822). Dies bringt Staat und Vigilanten in eine spezifische Beziehung zueinander, man könnte sogar sagen: Diese Konstellation bringt Vigilantismus erst hervor. Zugleich nimmt diese Beziehung, wie sich zeigen wird, unterschiedliche Formen an.

Die vorangegangenen Überlegungen bedeuten eine Öffnung der Perspektive und schließen an Einsichten der Soziologie der Gewalt an, welche die ambivalente Rolle von Gewalt für Soziales betonten. Gewalt ist „Teil der großen weltgeschichtlichen Ökonomie“, wie Popitz, Burckhardt zitierend, anmerkt (Popitz 1994, 55). Sie markiert und sichert Macht und soziale Ordnung ebenso, wie sie Macht und soziale Ordnung angreifen und zerstören kann. Dies gilt gleichermaßen für vigilante Akteure. Auch deren Handlungen stehen im Kontrast zu zugelassenen Formen der Gewaltanwendung, doch verfolgen sie oft das Ziel, soziale Ordnung wieder herzustellen bzw. zu bewahren (Kowalewski 2002, 426f.). Diese Uneindeutigkeit reproduziert sich gerade auch im Verhältnis vigilanter Gruppen oder Akteure zum Staat. Insofern würde in der Tat, wie Arfsten kürzlich anmerkte, „eine Herangehensweise, die von einem antagonistischen Verhältnis zwischen Vigilanten und Staat ausgeht, das Verständnis von Vigilantismus unnötigerweise limitieren“ (Arfsten 2012, 113). 


\section{Drei Typen von Vigilantismus}

Bislang existieren hauptsächlich zwei Vorschläge zur Typisierung des Vigilantismus. R.M. Brown nimmt hierfür eine eher historische Unterscheidung vor (Brown 1975). Die erste Form des Vigilantismus zielte demnach auf die Etablierung von ,law and order in den neuen Siedlungen des amerikanischen Westens und versuchte, quasi-staatliche Strukturen zu installieren. Die zweite Form, von Brown manchmal „new Vigilantism“ genannt, erweiterte ihre Ziele und richtete sich nun vermehrt gegen ganze Personengruppen religiöser, politischer oder ethnischer Art, die als Störung oder Bedrohung der etablierten Ordnung angesehen wurden. Dabei hatten diese Vigilanten vor allem eine ideale moralische Ordnung vor Augen, was sie auch gegen ,Trinker` und ihre Familien vernachlässigende Männer vorgehen ließ. Kurz gesagt, agierte die erste Variante des Vigilantismus an Stelle des Staates und seines Strafrechts, während die zweite Variante - trotz eines funktionierenden Rechtssystems - parallele Straf- und Rechtsnormen zu etablieren versuchte (Brown 1975, 123). Brown differenziert diese grundlegende Unterscheidung nicht weiter und gibt beiden Typen auch keine spezifischen Label. Zudem ordnet er sie je spezifischen historischen Phasen zu, sieht sie also eher als zeitlich aufeinander folgende Entwicklungsstufen denn als Spielarten, die auch parallel auftreten können.

Rosenbaum und Sederbergs Typologie umfasst dagegen drei Vigilantismus-Arten (Rosenbaum/ Sederberg 1975). Unterschieden werden diese anhand des jeweiligen ,Gegners‘. Variante 1 bezeichnen sie als crime control vigilantism, der sich gegen Personen richtet, denen Rechtsverletzungen unterstellt werden: Einbrecher, Drogenhändler, säumige Schuldner. Den zweiten Typus stellt der social group control vigilantism dar. Dabei geraten soziale Gruppen oder Einzelpersonen in den Fokus, die als kulturell abweichend und bedrohlich empfunden werden. Der dritte Typ schließlich, der regime control vigilantism, sieht im politischen Zentrum an sich die Hauptursache für gesellschaftliche Probleme. Dementsprechend streben die Vigilanten dieses Typs die Ablösung der jeweiligen Staatsführung an. Rosenbaum und Sederberg unterscheiden schließlich noch zwischen öffentlichen und privaten Akteuren und kommen damit im Ergebnis insgesamt zu sechs Varianten.[2]

Beide Vorschläge liegen nicht weit voneinander entfernt. Die jeweils ersten Typen ähneln sich stark in ihrer Orientierung auf - unterstellte - kriminelle Handlungen. Die jeweils zweiten Typen sind sich insofern nah, als bei beiden weniger klassische Kriminalität, sondern die wahrgenommene
[2] Der Vollständigkeit halber sei noch die Typologie von Martha Huggins (1991, 4ff.) erwähnt, die aber ohne analytischen Bezugspunkt eher eine Aufzählung unterschiedlicher Aktionsformen ist (bspw. lynchen, death squads etc.). 
Andersartigkeit von Gruppen und Personen das Problem ist, auf das die Vigilanten mit Gewalt antworten. Allein der dritte regimebezogene Typ bei Rosenbaum und Sederberg findet in Browns Arbeit kein Pendant.

Grundsätzlich halte ich beide Vorschläge für sinnvoll und hilfreich, wenn auch nicht für ausreichend. Browns Typisierung ist zu nah an konkreten historischen Kontexten und zu mechanisch gedacht; so scheint bei ihm der kriminalitätsbezogene Vigilantismus fast automatisch zu verschwinden, wenn einmal staatliche Strukturen etabliert sind. Die Unterscheidung von Rosenbaum und Sederberg nimmt zwar nicht solche Engführungen vor und erweitert die Reihe sinnvollerweise um einen dritten Typus. Ihre Entscheidung, die Typologie entlang der Problemlagen zu entwickeln, auf welche die Vigilanten reagieren, scheint mir jedoch vom theoretischen Gehalt zu schwach. Analytisch wird damit nicht vom Kern des Phänomens Vigilantismus Ausgang genommen. Dieser scheint mir eben gerade darin zu liegen, dass vigilante Akteure Gewalt anwenden und damit eine Kernkompetenz moderner Staaten beanspruchen, ohne selbst staatliche Akteure zu sein. Über den eigenen Einsatz von Gewalt wird damit unweigerlich ein Verhältnis zum Staat hergestellt, und zwar eines, das auf den ersten Blick konkurrierend bzw. antagonistisch aussieht - es aber keineswegs immer ist. Damit rückt ein Aspekt ins Zentrum, den die beiden anderen Typologien in gewisser Weise für gelöst halten. Immer, so deren Annahme, findet Vigilantismus jenseits staatlicher Strukturen statt, und muss so auch als Gegenentwurf zum Staat gedacht werden. Tatsächlich jedoch kann diese Perspektive differenziert werden.

Noch einmal anders gesagt: Das über den Gewalteinsatz hergestellte Verhältnis zum Staat, das damit quasi-staatliche Agieren von nicht-staatlichen Akteuren, scheint mir im Weberschen Sinne typisch für den Vigilantismus zu sein und damit als Referenzpunkt einer Typologie angemessener als die bisherigen Vorschläge. Wie angekündigt, wird deshalb die folgende Typologie entlang der Frage entwickelt: in welchem Verhältnis die vigilanten Akteure und deren Handlungen zum Staat stehen. Im Ergebnis dieser Überlegungen werde ich drei Typen des Vigilantismus unterscheiden: das Handeln an Stelle des Staates, das Handeln als besserer Staat und das Handeln jenseits des Staates.

Sicherlich sind andere Typologien denkbar. So könnte man vigilante Handlungen bspw. nach ihrer Dauerhaftigkeit analysieren und würde dann, vereinfacht gesagt, spontane und einmalige Aktionen (Lynchjustiz durch Mobs) von wiederholten und dauerhaft unternommenen Handlungen 
(organisierte Bürgerwehren) unterscheiden. Auch der Grad bzw. die Art der Organisiertheit selbst könnte als Kriterium einer Typisierung dienen. Die Typisierung entlang des jeweiligen Staatsbezuges scheint mir aber insofern sinnvoller, als sie gegenstandsbezogener ist - stellt die (mindestens implizite) Herausforderung des Staates doch ein zentrales Charakteristikum vigilanten Handelns dar.

\section{Typ I: Handeln an Stelle des Staates}

Der erste Typ von Vigilantismus umfasst Handlungen, die von nicht-staatlichen Akteuren an Stelle des Staates ausgeführt werden. Die Vigilanten dieses Typs agieren also gewissermaßen in Stellvertretung staatlicher Instanzen, da diese - aus welchen Gründen auch immer - im spezifischen Moment nicht vor Ort sind, nicht tätig werden können oder wollen.[3]

Ihnen geht es nicht primär darum, anders zu handeln als der Staat und seine Institutionen (Polizei), sondern eben an seiner Stelle. Oft geht es dabei um „Schutz“ und „Wehr“ des eigenen Besitzes, Territoriums oder Körpers. Dieser Schutz kann nicht vom Staat garantiert werden und wird deshalb selbst in die Hand genommen. Das eigene Handeln wird in diesen Fällen vorrangig als verteidigende Maßnahme legitimiert. Dies heißt auch, dass die Vigilanten dieses Typs nicht grundsätzlich das staatliche Gewaltmonopol bestreiten oder dauerhaft für sich beanspruchen würden. Sie agieren im Grunde nur temporär - eben bis der Staat wieder tätig wird.

Klassisches Beispiel für diese Art von Vigilantismus sind die von Brown beschriebenen Gruppen im Westen der Vereinigten Staaten, die an Stelle weit entfernter oder schlecht funktionierender staatlicher Instanzen bei der Verfolgung und Bestrafung von Viehdieben oder Schmugglern agierten (vgl. Brown 1975). In der Erklärung zu seiner Gründung stellte das San Francisco Committee of Vigilance von 1851 dementsprechend fest: „It has become apparent to the Citizens of San Francisco that there is no security for life and property, either under the regulations of society as it at present exists, or under the law as now administered; therefore, the citizens whose names are hereunto attached do unite themselves into an association for the maintenance of the peace and good order of society, and the preservation of the lives and property of the citizens of San Francisco, and do bind ourselves, each unto the other, to do and perform every lawful act for the maintenance of law and order, and to sustain the laws when faithfully and properly administered“ (zitiert in Abrahams
[3] Dies ist natürlich mindestens insofern problematisch, als es immer eine Unterstellung bleiben muss, dass die polizeilichen Behörden genauso vorgegangen wären. Als Kategorisierung dieses Handelns dient das Label „an Stelle des Staates" aber insofern, als vor allem der Staatsbezug der Vigilanten dieses Typs deutlich gemacht wird. Der Staat wird eben nicht als Konkurrenz, sondern primär als unzulänglich begriffen. 
1998, 58f.). Nicht die Gründung eines eigenen Staates oder die Einführung neuer Gesetze, sondern vielmehr die rechtmäßige Durchsetzung von bestehendem Recht und Gesetz war die Kernforderung dieser Gruppen; solange dies der Staat aber nicht gewährleisten konnte, übernahmen die vigilantes diese Aufgaben selbst. Offenkundig hatte der Vigilantismus hier deutlich organisierte Formen angenommen. Die Gruppen, die sich auch in der Nachfolge des San Francisco Committee of Vigilance gründeten, waren identifizierbare Akteure, die auf Dauer mit mehr oder minder eindeutigen Zielen und Mitgliedern bestanden. Dieser vergleichsweise hohe Organisationsgrad - das Committee hatte regelrechte Mitgliedsanträge - war sicherlich auch dem Umstand geschuldet, dass auf absehbare Sicht nicht mit einer höheren staatlichen Präsenz zu rechnen war.

Eine weniger organisierte Form eines solchen stellvertretenden Vigilantismus beschreibt Daniel Goldstein für Bolivien. Wiederholt wurden und werden dort (mutmaßliche) Einbrecher und Diebe von Dorfbewohnern nicht nur gestellt und festgehalten, sondern öffentlich gelyncht. Auch wenn diese Bestrafung in ihrer Härte weit über das hinausgeht, was staatliche Instanzen in Anwendung bringen würden, sehen die entsprechenden Gruppen sich selbst nicht als besseren Staat, sondern primär als Ersatz für eine sonst gänzlich fehlende Ordnungsmacht. „Vigilantism in this context may be understood as a political or moral statement that challenges state legitimacy and the official claim to a ,rule of law', not to overturn the state but, in classic Hobbesian fashion, to recall it to its legal obligations" (Goldstein 2003, 24f.).

Ähnliches ließ sich für die Amadlozi konstatieren, eine Gruppe aus den südafrikanischen Townships von Port Elizabeth. Sie bestand ausschließlich aus männlichen Bewohnern dieser Townships, die zwischenzeitlich sogar in uniformen T-Shirts auftrat, bei der Gewaltanwendung wenig Zurückhaltung an den Tag legte und sich vor allem der Diebstahlsbekämpfung und -bestrafung verschrieben hatte. „The state mattered for the Amadlozi. All its activities either mimicked the state's procedural forms in great detail or drew on its symbolic forms. When the Amadlozi challenged the state by issuing memoranda, the challenge confirmed the state and was directed at rectifying state practices and modes of being. Challenges were not aimed at undoing the state or establishing a different state: the aim was more state, not less" (Buur 2006, 750).[4]

Wenngleich auch solche Gruppen keine explizit anti-staatlichen Ziele verfolgen, ist ihr Agieren - aus Sicht des Staates - nicht legal und auch nicht risikolos. Denn keineswegs ist garantiert, dass sich das vigilante Handeln von Gruppen diesen Typs dauerhaft auf Stellvertretung beschränkt. Die
[4] Mitunter setzt der Staat auch vigilante Gruppen an seine Stelle bzw. fördert deren Aktivitäten. Dies war etwa bei der in Tansania tätigen sungusungu-Bewegung der Fall (vgl. Abrahams 1998; Heald 2005). 
autodefensas in Kolumbien sind ein gutes Beispiel dafür, dass anfangs noch spontan agierende Personen sich organisieren können und damit die Ausrichtung ihrer Gruppe nachhaltig verändern. Die autodefensas hatten ihren Ursprung in der eigenmächtigen Abwehr bzw. Bestrafung von Drogenbanden durch Bauern und Landbesitzer im südlichen Kolumbien (Fischer/Cubides 2000). Diese Aktivitäten waren anfangs noch spontan und unorganisiert. Daraus entwickelten sich eigene Milizen, die schließlich offizielle Anerkennung durch den Staat fanden, der sich selbst nicht in der Lage sah, ausreichend und effektiv gegen die Drogen-Guerilla vorzugehen. Bis zu diesem Stadium agierten die autodefensas also in deutlicher Stellvertretung, an Stelle des Staates. Dann allerdings weiteten diese Gruppen ihre Aktivitäten aus, wuchsen zahlenmäßig an und entwickelten sich zu regelrechten paramilitärischen Gruppen. Diese waren staatlicherseits keineswegs mehr einfach zu kontrollieren, beanspruchten die Kontrolle über ganze Territorien und stellten schließlich eine Art Staat im Staat dar - eine Form des Vigilantismus, die später als dritter Typ (,Handeln jenseits des Staates') beschrieben wird.

Zurück jedoch zum Vigilantismus an Stelle des Staates: Was lässt sich zu dessen politischer Relevanz sagen? Im Vergleich zu den beiden folgenden Vigilantismus-Typen ist der politische Charakter des stellvertretenden Vigilantismus gering(er) ausgeprägt. Dies ist insbesondere dort der Fall, wo man es mit eher unorganisierten und spontanen, aus momentanen Bedrohungssituationen heraus angewandter Gewalt bzw. eben spontanen Bestrafungsaktionen wie dem Lynchen auf frischer Tat gefasster Krimineller, zu tun hat. Solchen Aktionen liegt selten ein dezidiertes politisches Programm zu Grunde.

Allerdings hat allein schon die eigenmächtige Gewaltanwendung und damit der Bruch des staatlichen Gewaltmonopols zwangsläufig eine politische Dimension. Der Staat, wie schwach oder stark institutionalisiert auch immer, kann solche Handlungen nicht tolerieren bzw. ignorieren. Er muss sich zu ihnen verhalten. Die Art und Weise, wie er sich dazu verhält, hat wiederum politische Konsequenzen. Die vigilante Gewalt dieses Typs repräsentiert somit „,a simultaneous embrace and rejection of the official order; it reaffirms the power of the state to enforce the law, while at the same time suggesting that justice may be attained apart from the law" (Goldstein 2003, 25).

Zudem: Auch wenn der stellvertretende Vigilantismus ,nur' Rechtsdurchsetzung und nicht etwa Rechtsänderungen einfordert, stellt er eine Anklage an diesen Staat und damit eine politische Botschaft dar. Beklagt wird eben das Nicht-Funktionieren staatlicher Instanzen, die Abwesenheit der 
Polizei, die Inaktivität von Gerichten. Dies mag nicht immer explizit der Fall sein und kann natürlich von Fall zu Fall variieren, aber im Grunde wird diese moralisch-politische Anklage gegenüber dem nichts tuenden Staat immer mitgeführt. Damit geht dieses vigilante Handeln auch über das rein Defensive, das bloße Verteidigen hinaus - es wird vielmehr offensiv. Die politischen Implikationen dieses Typs sind somit, wenn auch indirekt, deutlich gegeben.

\section{Typ II: Handeln als der bessere Staat}

Ein zweiter Typ des Vigilantismus umfasst Handlungen, die ausgeführt werden, weil der Staat es nicht tun würde. Hier wird also nicht an Stelle des prinzipiell ebenso agierenden Staates gehandelt, sondern gewissermaßen neben dem Staat oder aus der Perspektive der Vigilanten: als besserer Staat. Dies muss keine grundsätzliche Infragestellung der Legitimität des Staates implizieren, richtet sich aber gegen die konkrete Praxis der staatlichen Rechtssetzung und des Strafens.

,Besser als der Staat' kann hier wiederum zweierlei bedeuten. Die erste Variante umfasst jene Fälle, in denen der Staat zwar Strafen für bestimmte Handlungen verhängt, diese aber seitens der Vigilanten als zu niedrig (zu wenig schwerwiegend, zu kurz) angesehen werden und deshalb eigenhändig verschärft werden. ,Verbessert' wird hier also das Verhältnis von unerlaubter Handlung und Sanktion; die Vigilanten agieren hier praktisch als ,härterer Staat‘.

Diesem Vigilantismus als besserer Staat entsprechen wiederum historische Beispiele aus den Vereinigten Staaten. Denn nicht alle Vigilanten handelten dort in Stellvertretung weit entfernter staatlicher Instanzen. Gerade den zu Beginn des 20. Jahrhunderts agierenden Gruppen ging es darum, anders und vor allem härter als die staatlichen Gerichte zu urteilen und zu bestrafen.[5] Dahinter standen insbesondere rassistische Motive. „In the eye of the mobs, the legal process was not just slow and uncertain; it was also too removed and too restrained. For these particular 'criminals', and for these particular 'crimes'. Southern mobs wanted to wreak their own brand of vengeance without intermediaries. The mode they preferred was impassioned, personalized, and communal; the techniques they utilized were deliberately cruel and unusual. Why? Because the crimes in question were perceived as crimes of lese majesty - challenges to the social order and the racial code upon which that order depended" (Garland 2005, 816).
[5] Brown fasst diese Gruppen, wie erwähnt, auch unter New Vigilantes. 
In der zweiten Variante rührt das vigilante Gewalthandeln aus dem Umstand, dass der Staat bestimmte Delikte nicht als Delikte, sondern als straffreie bzw. rechtlich zulässige Handlungen ansieht. Dies ist aus Sicht der privaten Gewaltakteure nicht hinnehmbar, und so agieren sie in diesen Fällen gewissermaßen als erweiterter Staat. Erweitert wird hier der Bereich sanktionierter Handlungen und aus dieser Perspektive die eigene Sicherheit, die öffentliche Moral oder das gemeinsame Zusammenleben, verbessert‘.

Kowalewski nennt die Phänomene, gegen die diese Art von Vigilantismus vorgeht, „kulturelle Devianz“: (unterstellte) Abweichungen von einer (wiederum unterstellten) richtigen und verbindlichen Lebensweise (vgl. Kowalewski 2002). Ein schon von Kowalewski angeführtes Beispiel und auch heute, etwa in Russland, beobachtbar, sind gewalttätige und organisierte Angriffe gegenüber Homosexuellen. Die Anlässe dafür sind unterschiedlich, die Zielrichtung aber ist in jedem Fall gleich: die Bestrafung bzw. Unterdrückung einer Lebensweise, die staatlicherseits nicht sanktioniert wird. Dem gleichen Muster folgen die Anschläge auf Abtreibungspraxen bzw. -ärzte in den USA, bei denen vor allem in den 1990er Jahren, zuletzt erst 2009, mehrere Mediziner ums Leben kamen oder schwer verletzt wurden.

Der Vigilantismus dieses Typs ist ein deutlich politischerer als der in Stellvertretung des Staates geschehende. Zum einen wird auch hier natürlich das staatliche Gewaltmonopol gebrochen und dies erkennbar offensiver als bei Typ I. Denn keineswegs erfolgen die vigilanten Aktionen aus dem Motiv des Schutzes oder der Verteidigung heraus. Vielmehr wird hier Gewalt angewandt, um eigenmächtig Recht zu sprechen und insbesondere Verhalten zu bestrafen, das staatlicherseits toleriert wird. Letztlich zielen damit die hier beobachtbaren Taten nicht auf die generelle Auflösung geltenden Rechts, sondern vielmehr auf dessen Transformation. Recht und Bestrafung sollen entweder verschärft oder auf neue Bereiche ausgedehnt werden. Solange dies nicht der Fall ist, nehmen die Handelnden das (erhoffte) Recht selbst in die Hand. Anders gesagt: Der Staat soll weiter strafender und Recht setzender Akteur sein, er soll seine Praxis aber in spezifischen Punkten ändern. Dies ist unverkennbar auch ein politisch-moralisches Signal an die jeweilige Gesellschaft, ihre mehrheitliche Meinung zu ändern.

Besonders deutlich werden die politischen Implikationen dieses Vigilantismus-Typs an Beispielen aus Lateinamerika. Die dortigen Fälle gehen nicht nur über das staatlicherseits übliche Maß an Bestrafung hinaus und münden nicht selten in der Tötung der Beschuldigten. Vielmehr sind sie 
zudem oftmals von expliziten politischen Protesten und Demonstrationen begleitet. Sie sind so „in part a politically charged expression of popular will through violence, aimed squarely at outsiders and the state" (Snodgrass Godoy 2004, 638).

Die Legitimationen sind dabei sehr heterogen und reichen von der Berufung auf „wirkliche Gerechtigkeit“ bis zum Verweis auf bindende Tradition. Gemein ist diesen Varianten, dass das staatliche (Nicht)Handeln immer unter Bezug auf eine höhere und als wichtiger erachtete Instanz oder Idee bewertet wird, etwa Gerechtigkeit, Tradition oder Ehre. Obwohl es also beispielsweise ,gerecht wäre, kommt ein Betrüger - etwa aufgrund von Verfahrensfehlern - ungestraft davon. Der Staat will hier also offensichtlich nicht tätig werden - deshalb wird man es selbst.

Auch wenn etliche der genannten Beispiele dies nahelegen: Keineswegs muss dieser zweite Typ des Vigilantismus immer organisiert auftreten. Gewalthandeln „als besserer Staat“ findet auch unorganisiert statt. In diesen Fällen hat es dann eher konkrete bzw. spezifische Anlässe und Ursachen, wird zudem eher von Einzelpersonen als ganzen Gruppen unternommen. Prominentes Beispiel für diese Spielart des Vigilantismus ist etwa Marianne Bachmeier, die 1981 im Gerichtssaal den mutmaßlichen Mörder ihrer Tochter erschoss. Dies ist nur ein Fall von vielen ähnlich gelagerten Konstellationen, wo aus der Haft Entlassene (oder nicht Verurteilte) von den Hinterbliebenen der Opfer durch Gewaltakte unterschiedlicher Art nochmals (oder eben erstmals) bestraft werden. Die Täter handeln hier nicht in Stellvertretung des Staates, denn dieser ist ja zuvor tätig gewesen - allerdings mit einem für die Vigilanten unbefriedigenden Ergebnis. Deshalb werden sie selbst tätig und sorgen (aus ihrer Perspektive) für ,wahre` oder ,echte‘ Gerechtigkeit.[6]

\section{Typ III: Handeln jenseits des Staates}

Dieser dritte Typ schließlich ist weder bei Rosenbaum und Sederberg, noch bei Brown systematisch herausgearbeitet worden, obwohl er über verschiedene Fallstudien, aber auch in der Arbeit von Abrahams durchaus Berücksichtigung gefunden hat. Diese Form von Vigilantismus strebt die gewaltsame Etablierung von ganz eigenen sozialen Ordnungen an - jenseits des Staates. Hier soll weder an Stelle eines als legitim angesehenen Staates wie im Typ I, noch in Verlängerung oder Verschärfung seiner Kompetenzen wie in Typ II gehandelt werden. Vielmehr geht es in diesen Beispielen um die Etablierung von Räumen eigenen Rechts und eigener Legitimität. Die Akteure legen
[6] Man kann hier einwenden, dass solche Beispiele eher als ,Rache' zu verstehen seien (vgl. Paul 2005). Das mag für etliche Fälle und insbesondere das Beispiel Bachmeiers zutreffen. Zugleich sind aber nicht-organisierte Taten ,als besserer Staat' auch dort beobachtbar, wo keine Schädigung des Täter oder Tätergruppe vorausging. Das Konzept der Rache scheint mir darum gewissermaßen quer zu dem des Vigilantismus zu liegen. Rache wäre so als reaktive Form der gewaltsamen Konfliktregulierung zu verstehen, während Vigilantismus auch vorbeugend geschehen kann und zudem nicht das eigene Betroffensein zur Bedingung hat. So gesehen, handelt es sich nicht um konkurrierende Konzepte, und je nach Perspektive oder jeweiligem Erkenntnisinteresse mag es in konkreten Fällen sinnvoller sein, von Rache oder Vigilantismus zu sprechen, ohne dass beide Lesarten sich wechselseitig ausschließen müssten. 
hier grundsätzlich neu fest, wer was zu tun und zu lassen hat, was Recht und was Unrecht ist - und wollen diese Kompetenz auch nicht an einen Staat abgeben. Dieser Typ von Vigilantismus ist häufig für Regionen beschrieben worden, in denen staatliche Herrschaft schwach ausgeprägt ist. Beispiele hierfür sind die als „brown areas“ beschriebenen Gebiete in Lateinamerika oder afrikanischen Staaten, die sich gänzlich staatlicher Kontrolle entziehen (vgl. Trotha 1995; Waldmann 1997). Dies mögen ländliche Gebiete sein, aber auch sogenannte städtische Elendsviertel.

Jenseits dieser Gemeinsamkeit changieren der Charakter der jeweiligen Gruppen, ihre innere Struktur und ihre Zielsetzungen recht stark. Mitunter sind die Vigilanten dieses Typs bewaffnete Banden und Milizen, die in Gebieten die Kontrolle über die öffentliche Ordnung übernommen haben, die vom Staat schlichtweg nicht mehr gewährleistet wird. Bei anderen Gruppen sind deutlichere politische Ziele im Spiel; deren Vigilantismus ist organisierter, dauerhafter und strebt nach einer stabileren Existenz jenseits des jeweiligen Zentralstaates. Die „gewaltsame Selbsthilfe wird [dort] als Weg der Befreiung von Unterprivilegierung, Ausbeutung und Elend gedacht“" (Trotha 1995, 143). Beispiel für eine solche vigilante Gruppierung, die tatsächlich (weitestgehend) jenseits des Staates agiert, ist der Oodua People's Congress (OPC) in Nigeria. Er gründete sich 1994 als eine Art „ethno-regional grassroots movement“, um die Interessen der nigerianischen Yoruba gegenüber der repressiven Zentralregierung besser durchzusetzen. Bald nach seiner Gründung militarisierte sich der OPC merklich und wurde die primäre Ordnungsmacht in weiten Teilen der von den Yoruba bewohnten Teile des Landes. „It became an informal law-enforcement agency, providing security in areas deserted by the police, chasing and - gruesomely - eliminating armed robbers, settling disputes, acting as debt-collector etc.“ (Guichaoua 2009, 530). Ziel der Aktivitäten war aber nicht, den Staat in den entsprechenden Gebieten wieder in Verantwortung zu bringen, wie es bei den beiden zuvor besprochenen Vigilantismus-Typen der Fall wäre: „OPC's violence had two main targets, the non-Yoruba populations said to illegitimately occupy the Yoruba territory and the Federal Police, incarnating the oppression of the Centre" (ebd.).[7] Eine ähnliche Karriere durchliefen die kolumbianischen autodefensas, die bereits beim Typus des stellvertretenden Vigilantismus beschrieben wurden. Als eine solche Gruppe beginnend, entwickelten sie sich zu regelrechten paramilitärischen Einheiten, die Territorien ganz eigener Ordnung errichteten und somit zunehmend in Konfrontation zum Staat traten.[8]
[7] Solche Fälle sind auch nicht mit Rechtspluralismus zu verwechseln. In den gängigen Fällen von Rechtspluralismus geht es ja um ein Nebeneinander von unterschiedlichen Rechtsformen, die jede für sich spezifische Legitimität haben, und deren Geltung dann fallspezifisch ausgehandelt bzw. festgelegt wird. Keinesfalls hat man es dort mit exklusiv geltendem Recht zu tun, das gegenüber anderen Rechten Vorrang hätte (vgl. Benda-Beckmann 2008).

[8] Äquivalente in deutlich kleinerem Maßstab in westeuropäischen Ländern sind Gebiete, in denen Rockerbanden versuchen, den Zugriff auf Recht und Ordnung primär unter ihre Kontrolle zu bekommen. 
Die Veränderung im Charakter dieser Gruppierungen geht nicht zuletzt mit einer Veränderung ihrer Organisationsformen einher. Diese werden hierarchischer, klarer strukturiert und bekommen deutlich erkennbarere Grenzen bzw. regelrechte Mitglieder. Generell lässt sich sagen, dass die Vigilanten des Typs III einen zweifellos höheren Organisationsgrad als viele der Beispiele aus Typ I und Typ II erkennen lassen. Dies ist kein Zufall, sondern hängt systematisch mit einem anderen Bezug zum Staat zusammen. Ein Einzeltäter oder eine lose zusammen geschlossene Gruppe wird kaum den Anspruch erheben (können), auch nur halbwegs dauerhaft einen Raum eigenen Rechts jenseits des Staates zu etablieren. Dafür erfordert es nicht nur ein Mehr an Ressourcen, sondern eben auch einen deutlich höheres Maß an Organisiertheit.

Die zuvor genannten Beispiele erinnern in mancher Hinsicht an Phänomene, die unter Begriffen wie armed groups, Warlords oder Rebellen verhandelt werden. So schwer sich dies pauschal sagen lässt: Am ehesten sehe ich vigilante Aktivitäten bei Warlords bzw. einigen der armed groups (vgl. die Beispiele bei Schlichte 2009, 30ff.), die nicht primär das jeweilige politische Zentrum des Landes im Ziel haben, sondern sich mit der Kontrolle über spezifische Landesteile ,begnügen‘. Mittel der Kontrolle ist eben auch die Errichtung eigener Rechtsräume, auch wenn diese häufig von mehr oder minder willkürlicher Gewaltausübung geprägt sein können. Rebellische Gruppen dagegen übernehmen zwar auch die Kontrolle über „eigene“ Gebiete. Ziel ist es aber zumeist, diese nicht jenseits des Staates zu etablieren, sondern diese Gebiete zu erweitern und den Staat als solchen anzugreifen bzw. zu übernehmen. Politische Interessen sind hier noch einmal deutlicher ausgeprägt. Vigilante Aktivitäten sind damit zwar nicht gänzlich ausgeschlossen, aber doch weniger dominant.

Zugleich darf man sich den Vigilantismus jenseits des Staates nicht zu geordnet vorstellen: Terror und Massaker sind oftmals quasi institutionalisiert (Trotha 1995, 143) und an der Tagesordnung, und sie verlaufen nicht selten willkürlich; die Grenzen zwischen staatlicher Justiz und dem kriminellen Sektor werden mehr und mehr unklar. Eine Rede vom ,state within a state hat hier somit ihre offensichtlichen Grenzen. Beispielhaft lässt sich das wiederum an einem Beispiel aus Nigeria zeigen. Dort wurden 1998 die Bakassi Boys gegründet; anfänglich eine Gruppe von jungen Männern, die von lokalen Händlern in Aba dafür bezahlt wurden, den lokalen Markt zu bewachen und Kriminelle zu verfolgen. Die Bakassi Boys machten schnell durch ein sowohl effektives wie brutales Vorgehen von sich reden - öffentliche Hinrichtungen waren dabei eingeschlossen. Schon nach wenigen Jahren hatten sie in weiten Teilen des südöstlichen Nigerias eine Art Gewaltmonopol und das unter 
Duldung lokaler Politiker. „The state governors offered official backing to the vigilantes, giving them formal names (the Abia State Vigilante Services and Anambra Vigilante Services), funding, vehicles, and political cover" (Smith 2004, 431). Mehr und mehr jedoch gerieten die Bakassi Boys in Misskredit. Offenkundig traten sie häufig nicht nur beschützend oder in Reaktion auf stattgefundene kriminelle Handlungen auf den Plan, sondern verfolgten ganz eigene Ziele, wobei sie regelmäßig Gewalt gegen Unschuldige bzw. zum eigenen Vorteil an wandten (Meagher 2007). Der zunehmend aufkommende Verdacht, selbst korrupt und kriminell zu sein, ließ sie entscheidend an Anhängerschaft und Rückhalt verlieren. Im Jahr 2002 wurden sie durch staatliche Truppen weitestgehend zerschlagen.

Politisch sind die Vigilanten jenseits des Staates also insofern, als sie in deutlicher Frontstellung zum Staat auftreten. Weder ein staatliches Gewaltmonopol, noch dessen Anspruch auf Rechtssetzung und Bestrafung wird in diesen Beispielen als legitim anerkannt. Beides soll komplett abgelöst bzw. jenseits der Geltung des staatlichen Bereiches soll eine eigene Rechts- und Gewaltordnung geschaffen werden. Der Zentralstaat - wie stark oder schwach auch immer ausgeprägt - ist hier nicht der eigentliche Adressat des politischen Handelns dieser Gruppen; sie betreiben gewissermaßen Politik auf eigene Rechnung, sie setzen eher auf Separierung und Abspaltung denn auf Veränderung oder Einmischung. Die Herausforderung für den solche Territorien eigentlich beanspruchenden Zentralstaat ist damit eine andere als im Typ II. Er muss sich hier nicht als richtig handelnder Akteur beweisen und legitimieren, sondern überhaupt erst (wieder) in Erscheinung treten und Legitimität erlangen.

\section{Fazit}

Die hier entworfenen drei Spielarten des Vigilantismus sind idealtypisch zu verstehen. So gibt es etwa bei den Beispielen des Typ III zahlreiche Unschärfen, was deren Verhältnis zum Staat angeht. Ein Vigilantismus, der tatsächlich ,jenseits des Staates ' agiert, ist in der Realität sehr selten zu beobachten. Stacey Hunt weist in ihrer Analyse paramilitärischer Gruppen darauf hin, dass solche Gruppen keineswegs immer sauber von staatlichen Institutionen zu trennen sind. Sie sieht die von ihr analysierten Vigilanten vielmehr als eine Art Hybrid zwischen Staat und Beherrschten: „This ongoing process of state construction involves citizens in a dialectic of state and citizen formation, that 
transcends understandings of the paramilitaries as either tools of a terrorist state or violent eruptions of civil society that besiege the state" (Hunt 2009, 66). Insofern ist die Typologie auch Anlass grundsätzlich weiter und wieder über das Verhältnis von staatlichen und nicht-staatlichen Akteuren nachzudenken, wechselseitige Inanspruchnahmen und Imitationen zu analysieren und nicht sofort - ungeachtet des analytischen Ertrags der Kategorien - Trennungen beider Seiten vorzunehmen (ähnlich auch Arfsten 2012).

Schaut man sich andere Beispiele vigilanter Gruppen an, stellen diese nicht selten auch ,Mischungen' der hier entworfenen Typen dar. So werden mitunter Personen oder Gruppen staatlicherseits als Stellvertreter eingesetzt oder toleriert, um in einem spezifischen Gebiet an Stelle des Staates die Gewaltkontrolle auszuüben. In der Praxis agieren sie dann (auch) als Typ II, weil sie mitunter härter vorgehen, als der Staat es tun würde. Eine solche Gruppe sind etwa die Neu-Kosaken im Süden Russlands. Diese Wiedergründung einer ethnisch-militanten Gruppierung formierte sich seit den 1990er Jahren in der Tradition alter kosakischer Kampfverbände und verbindet nationale Folklore mit dem gewaltsamen Vorgehen gegen alles Nicht-Russische. Immer wieder werden diese Verbände dabei vom russischen Staat unterstützt oder sogar gezielt, etwa gegen Migranten, eingesetzt, sind zugleich jedoch nur bedingt zu kontrollieren (vgl. Derlugian/Cipko 1997).

Diese Einschränkungen sind aber zugleich ganz im Sinne des Hauptzieles der Typologie: Unterscheidungen im Feld des Vigilantismus vorzunehmen, um systematische Differenzen deutlich zu machen. Im Ergebnis können tatsächlich viele Beispiele aus dem Feld des Vigilantismus als politische Gewalt verstanden werden. Am deutlichsten ist dies sicherlich beim Vigilantismus des Typ II. Er sendet häufig explizit politische Signale an den Staat, seine Rechts- und Strafpraxis zu ändern. Schwierig zu charakterisieren sind die Fälle dieses Typs, weil sie noch immer ein relativ weites Feld an Motiven und Zielrichtungen umfassen: von denen, die schon als strafwürdig eingestufte Vergehen noch härter bestrafen wollen bis hin zu jenen, die noch ganz andere Dinge bestraft sehen wollen. In allen Fällen aber erkennen damit die Vigilanten die Kompetenzen des Staates an. Für diesen wiederum ist damit gerade diese Form des Vigilantismus kein einfaches Problem.

Dies wird vor allem im Vergleich deutlich. Der Typ I greift den Staat nicht oder kaum an, sondern agiert ,nur' in Stellvertretung; er stellt damit nur ein vorübergehendes Problem dar, das aber keine grundsätzlichen Maßnahmen oder Argumentationen erfordert. Beim Vigilantismus des Typ III ist die Konstellation insofern einfach, als diese Gruppen in klarer Gegnerschaft zum Staat stehen; 
Verhandlungsspielraum gibt es hier kaum, Zugeständnisse sind im Grunde ausgeschlossen, ohne die eigene grundsätzliche Position ernsthaft zu gefährden. Mit dem Vigilantismus dagegen, der sich als besserer Staat darstellt (Typ II), muss hingegen gestritten werden. Dies ist in der Frage der Gewaltanwendung noch ,leicht'. Wenn es aber um die inhaltlichen Forderungen bzw. Problemanzeigen geht (Umgang mit Homosexualität; Legalisierung von Abtreibungen etc.), sind dies auch jenseits des gewaltsamen Protestes Themen, die gesellschaftlich diskutiert werden, und auf die der Staat antworten muss. Der Einsatz von Gewalt delegitimiert auf der einen Seite solche politischen Forderungen (oder Proteste), er verleiht ihnen zugleich aber auch Nachdruck.

Vigilantismus ist aber eben nicht per se politische Gewalt. Dies trifft vor allem auf jene Fälle $\mathrm{zu}$, die sich dem Typ I zuordnen lassen, und dort wiederum auf diejenigen, die eher unorganisiert und von Einzelpersonen verübt werden. Hier geht es häufig um den situativ vorgenommen Schutz von Eigentum, das Stellen von (mutmaßlichen) Einbrechern bzw. Dieben. Politische Forderungen verbinden sich damit nicht unbedingt. Gleichwohl sind solche Fälle natürlich politisierbar. Nicht selten schließen sich an Fälle, in denen Personen verurteilt werden, weil sie eigenmächtig Gewalt gegenüber scheinbaren Straftätern anwandten, öffentliche Debatten um Ordnung, Sicherheit und das Funktionieren von Polizei und Justiz an. Ebenso gilt dies für Fälle des Typs II.

Solche Unterschiede nicht nur aufzuzeigen, sondern sie auch auf typische Eigenheiten des Phänomens Vigilantismus zurückführen zu können, ist aus meiner Sicht wichtiger Ertrag der hier vorgeschlagenen Typologie. Auch wenn dies sicherlich noch nicht erschöpfend geschah, konnte dies in ähnlicher Weise für die unterschiedliche Organisiertheit vigilanter Akteure gezeigt werden. Hier war bspw. deutlich erkennbar, dass gerade die Vigilanten des Typs III organisiert sind - und dies auch sein müssen, um tatsächlich jenseits des Staates eigene Rechtsräume zu schaffen. Diese Dauerhaftigkeit und Stabilität streben die Vigilanten, die an Stelle bzw. als besserer Staat agieren, nicht unbedingt an. Da sie jeweils ,nur' in Stellvertretung bzw. ergänzend, nicht aber in explizit konträrer Haltung zum Staat agieren, sind sie nicht unbedingt auf einen hohen Organisationsgrad angewiesen.

Eine solche Typologie ermöglicht es zudem, beobachtbare Transformationen und Veränderungen von vigilanten Akteuren systematisch erkennen zu können. Die Beispiele der autodefensas und der Bakassi Boys und ihre sich verändernden Ausrichtungen lassen sich nun nicht nur beschreiben, sondern präzise und theoretisch gehaltvoll rekonstruieren. Ihre Interpretation erfolgt damit vor dem Hintergrund einer Typologie, die - mit dem (jeweils unterschiedlichen) Bezug auf den 
Staat, ohne selbst Staat zu sein - ein typisches und zentrales Merkmal eines jeden Vigilantismus verdeutlicht und zum analytischen Fluchtpunkt wählt. Neben dem Beitrag zur Erforschung politischer Gewalt trägt dies auch ganz grundsätzlich zum weiteren Verständnis eines Phänomens bei, das zwar als ,eigentlich überwunden' gilt, tatsächlich aber auch von der Moderne - vielleicht unausweichlich - mitgeführt wird.

\section{Bibliographie}

Abrahams, R. (1998) Vigilant Citizens. Vigilantism and the State. Cambridge: Polity Press.

Arfsten, K.-S. (2012) 'Every man is entitled to defend his castle...'. Vigilantismus während der London 'riots'. In: Kriminologisches Journal 44 (2): 101-117.

Benda-Beckmann, F. (2008) Pluralismus von Recht und Ordnung. In: Behemoth. A Journal on Civilisation 1: 58-67.

Brown, R.M. (1975) Strain of Violence. Historical Studies of American Violence and Vigilantism. Oxford/New York: Oxford University Press.

Buur, L. (2006) Reordering Society. Vigilantism and Expressions of Sovereignty in Port Elizabeth's Townships. In: Development and Change 37 (4): 735-757.

Derlugian, G. M./Cipko, S. (1997) The Politics of Identity in a Russian Borderland Province. The Kuban Neo-Cossack Movement 1989-1996. In: Europe-Asia Studies 49 (8): 1485-1500.

Fischer, Th./ Cubides, F. (2000) Paramilitarismus in Kolumbien. Von der Privatjustiz zum politischen Akteur? In: Fischer, T./Krennerich, M. (Hg.) Politische Gewalt in Lateinamerika. Frankfurt/M.: Vervuert Verlag.

Garland, D. (2005) Penal Excess and Surplus Meaning. Public Torture Lynchings in TwentiethCentury America. In: Law \& Society Review 39 (4): 793-833.

Goldstein, D. M. (2003) 'In our own hands'. Lynching, Justice, and the Law in Bolivia. In: American Ethnologist 30 (1): 22-43.

Guichaoua, Y. (2009) Self-Determination Group or Extra-Legal Governance Agency? The Multifaceted Nature of the Oodua People's Congress in Nigeria. In: Journal of International Development 21: 520-533. 
Heald, S. (2005) State, Law, and Vigilantism in Northern Tanzania. In: African Affairs 105 (419): 265-283.

Heitmeyer, W. et el. (Hg.) (2002) Internationales Handbuch der Gewaltforschung. Wiesbaden: Westdeutscher Verlag.

Huggins, M. K. (1991) Introduction. Vigilantism and the State: A Look South and North. In: diess. (Hg.) Vigilantism and the State in Modern Latin America. New York: Praeger.

Hunt, S. (2009) Rethinking State, Civil Society and Citizen Partizipation. The Case of the Colombian Paramilitaries. In: Behemoth. A Journal on Civilization (1): 64-87.

Johnston, L. (1996) What is Vigilantism? In: British Journal of Criminology 36: 220-236.

Jones, A. (2004) Parainstitutional Violence in Latin America. In: Latin American Politics \& Society $46(4): 127-148$.

Kowalewski, D. (2002) Vigilantismus. In: Heitmeyer, W. al el. (Hg.) Internationales Handbuch der Gewaltforschung. Wiesbaden: Westdeutscher Verlag.

Meagher, K. (2007) Hijacking civil society: the inside story of the Bakassi Boys vigilante group of south-eastern Nigeria. In: Journal of modern African studies 45 (1): 89-115.

Paul, A. (2005) Die Rache und das Rätsel der Gabe. In: Leviathan 33 (2): 240-256.

Popitz, H. (1992) Phänomene der Macht. Tübingen: Mohr Siebeck.

Rosenbaum, H.J./Sederberg, P.C. (1976) Vigilantism: An Analysis of Establishment Violence. In: diess. (Hg.) Vigilante Politics, Pennsylvania: University of Pennsylvania Press.

Schlichte, K. (2009) In the Shadow of Violence. The Politics of Armed Groups. Frankfurt/M., New York: Campus.

Schmidt-Lux, Th. (2012) Vigilantismus. Ein Phänomen der Grenze? In: Kriminologisches Journal 44 (2): 118-132.

Smith, D. J. (2004) The Bakassi Boys. Vigilantism, Violence, and Political Imagination in Nigeria. In: Cultural Anthropology 19 (3): 429-455.

Snodgrass Godoy, A. (2004) When 'Justice' is Criminal. Lynchings in Contemporary Latin America. In: Theory and Society 33 (6): 621-651.

Trotha, T. v. (1995) Ordnungsformen der Gewalt oder Aussichten auf das Ende des staatlichen Gewaltmonopols. In: Nedelmann, B. (Hg.) Politische Institutionen im Wandel. Opladen: Westdeutscher Verlag: 129-165. 
Waldmann, P. (1997) Veralltäglichung von Gewalt. Das Beispiel Kolumbien. In: Trotha, T.v. (Hg.) Soziologie der Gewalt. Sonderheft 37 der Kölner Zeitschrift für Soziologie und Sozialpsychologie:141-161.

Weber, M. (1972) Wirtschaft und Gesellschaft. Tübingen: Mohr Siebeck. 